\title{
POWER ROOTS OF POLYNOMIALS
}

\section{JOHN BORIS MILLER}

Sufficient conditions are given for the existence of an $m$ th power root of one polynomial modulo another, over the complexes or the reals. Examples show the non-necessity of the conditions. In particular cases there can exist infinitely many square roots.

Let $K[\lambda]$ denote as usual the algebra of all polynomials in an indeterminate $\lambda$ over a field $K$. If $p(\lambda)$ and $f(\lambda)$ belong to $K[\lambda], m \in N$ and

$$
(p(\lambda))^{m}=f(\lambda)
$$

we say that $p(\lambda)$ is a power root of $f(\lambda)$, more precisely an $m$ th root.

It is not difficult to show that if this equation, with $f(\lambda)$ given in $C[\lambda]$, has any solutions $p(\lambda)$ then it has precisely $m$ solutions in $\mathbb{C}[\lambda]$. For suppose $f(\lambda)$ is monic (that is, has leading coefficient 1 ); then one verifies by solving for the coefficients of $p(\lambda)$ that there exists at most one solution $p(\lambda)$ which is monic; the general statement can be deduced from this.

Of more interest and abundance than power roots of polynomials are power roots of residue classes of polynomials. For any $w(\lambda) \in K[\lambda]$ let

$$
\mathfrak{U}_{w, K}:=K[\lambda] \bmod w(\lambda)
$$

denote the residue-class algebra over $K$ of $K[\lambda]$ modulo the principal ideal generated by $w(\lambda)$; its elements will be written $[f],[p], \ldots$ An $m$ th root of $[f]$ is any coset $[p]$ such that $[p]^{m}=[f]$. Our principal result is:

THEоReM. In the complex residue-class algebra $\mathfrak{U}_{w, C}$ where degree $(w) \geqslant 1$, a sufficient condition for a class $[f]$ to possess $m$ th roots of all orders $m \in \mathbb{N}$ is: that $f(\lambda)$ does not vanish at any multiple zero of $w(\lambda)$. In the real residue-class algebra $\mathfrak{U}_{w, \mathbb{R}}$, sufficient conditions are: that $w(\lambda)$ is real with real roots, $f(\lambda)$ is a real polynomial, $f(\lambda)$ does not vanish at any multiple zero of $w(\lambda)$, and $f(\lambda) \geqslant 0$ at every zero of $w(\lambda)$.

The proof of the theorem will be separated into the following two lemmas, whose proofs use the existence of $m$ th roots in algebras of matrices.

Received 17 February 1992

Copyright Clearance Centre, Inc. Serial-fee code: 0004-9729/93 \$A2.00+0.00. 
Lemma 1. With $K=\mathbb{C}$, let $m, n \in N, m \geqslant 2$, and suppose that in $\mathbb{C}[\lambda]$ :

(i) $w(\lambda)$ is a polynomial of degree $n$;

(ii) $f(\lambda)$ is a polynomial such that $f(b) \neq 0$ for each multiple zero $b$ of $w(\lambda)$. Then there exist polynomials $p(\lambda)$ and $q(\lambda)$ in $C[\lambda]$ such that

$$
(p(\lambda))^{m}=f(\lambda)+w(\lambda) q(\lambda) .
$$

Proof: The case $n=1, w(\lambda)=\lambda-b$ say, is disposed of by dividing $f(\lambda)$ by $\lambda-b$ to get $f(\lambda)=-(\lambda-b) q(\lambda)+a$ say, $a \in \mathbb{C}$, and taking $\operatorname{deg}(p)=0, p(\lambda)=a^{1 / m}$.

Henceforth suppose that $n \geqslant 2$ and that $\operatorname{deg}(f) \geqslant 1$. Let $b_{1}, b_{2}, \ldots, b_{r}$ be an enumeration of the distinct zeros of $w(\lambda)$, and let $k_{1}, k_{2}, \ldots, k_{r}$ be their multiplicities, so that $\sum_{j=1}^{r} k_{j}=n$ and

$$
w(\lambda)=\left(\lambda-b_{1}\right)^{k_{1}}\left(\lambda-b_{2}\right)^{k_{2}} \ldots\left(\lambda-b_{r}\right)^{k_{r}}
$$

(without loss of generality we assume that $w(\lambda)$ has leading coefficient 1 ). Let

$$
J_{k}(b)=\left(\begin{array}{llllll}
b & 1 & & & \\
& b & & & \\
& & \cdot & & & \\
& & & \cdot & & \\
& & & & b & 1 \\
& & & & b
\end{array}\right)
$$

denote the usual $k \times k$ Jordan block matrix, and let $W$ be the $n \times n$ block diagonal matrix

$$
W=\operatorname{diag}\left(J_{k_{1}}\left(b_{1}\right), J_{k_{2}}\left(b_{2}\right), \ldots, J_{k_{r}}\left(b_{r}\right)\right)
$$

The elementary divisors of $\boldsymbol{W}$ over $\mathbb{C}$ are

$$
\left(\lambda-b_{1}\right)^{k_{1}},\left(\lambda-b_{2}\right)^{k_{2}}, \ldots,\left(\lambda-b_{r}\right)^{k_{r}},
$$

and the minimal (annihilating) polynomial of $W$ is our given polynomial $w(\lambda)$ in (2).

Using the other given polynomial $f(\lambda)$ we have

$$
f(W)=\operatorname{diag}\left(f\left(J_{k_{1}}\left(b_{1}\right)\right), f\left(J_{k_{2}}\left(b_{2}\right)\right), \ldots, f\left(J_{k_{r}}\left(b_{r}\right)\right)\right) \text {. }
$$

Here well-known calculations give

$$
f\left(J_{k}(b)\right)=\left[f(b), \frac{f^{\prime}(b)}{1 !}, \ldots, \frac{f^{(k-1)}(b)}{(k-1) !}\right]
$$


that is, $f\left(J_{k}(b)\right)$ is the $k \times k$ upper triangular matrix with constant diagonals having for its top row the tuple shown in (7).

The matrix $f(W)$ in (6) has at least one matrix $m$ th root, that is, a solution $Y$ in $\mathfrak{M}_{n}(C)$ (the algebra of all $n \times n$ matrices over $C$ ) of the equation

$$
Y^{m}=f(W)
$$

namely

$$
Y=\operatorname{diag}\left(X_{1}, X_{2}, \ldots, X_{r}\right)
$$

where for each $j$,

$$
X_{j}^{m}=f\left(J_{h_{j}}\left(b_{j}\right)\right)
$$

Clearly, if an $X_{j}$ exists for each $j$, then (8) follows easily from (6), (9) and (10j).

The existence of $X_{j}$ is shown by its construction. See Gantmacher [1, pp.231-234] for the construction of square roots of matrices. For completeness we give a construction here, but prefer to rely upon the general functional calculus in the complex Banach algebra $\mathfrak{M}_{n}(\mathbb{C})$ with any algebra matrix norm (see [2, Theorem 5.2.5, pp.168-169]), since this method makes clear a commutativity property needed presently.

Assume first that $f\left(b_{j}\right) \neq 0$. Let $\rho$ be any ray from the origin in the complex plane, not passing through any nonzero values among the numbers $b_{j}, f\left(b_{j}\right)$ for $j=$ $1,2, \ldots, r$. Let $\Gamma(\mu)$ denote a small positively oriented circle about $\mu$ not intersecting $\rho$. Let $h$ denote any holomorphic branch function of the root relation $\lambda^{1 / m}$ on the plane cut along $\rho$ from 0 to $\infty$. Fix $j$; we shall suppress the suffix $j$ temporarily. The matrix

$$
X:=\frac{1}{2 \pi i} \int_{\Gamma(f(b))} h(\lambda)\left(\lambda I-f\left(J_{k}(b)\right)\right)^{-1} d \lambda
$$

is well defined, and if $l(\lambda):=\lambda^{m}$ then $[2$, Theorem 5.3.2, p.171]

$$
X^{m}=l(X)=l \circ h\left(f\left(J_{k}(b)\right)\right)=f\left(J_{k}(b)\right)
$$

Thus for each $j$ the matrix $X_{j}=X$ in (11) gives a solution of $(10 j)$.

Suppose instead that $f\left(b_{j}\right)=0$; by (ii) $k_{j}=1$, so $(10 \mathrm{j})$ in this case becomes $X_{j}^{m}=O$ in $\mathfrak{M}_{1}(\mathbb{C})$ and we therefore take $X_{j}=O$. (If $k>1$ then $J_{k}(0)$ has no $m$ th root, so we exclude this possibility.)

Definition (11) shows that $X$ belongs to the second commutant of $f\left(J_{k}(b)\right)$, that is, it commutes with every matrix which commutes with $f\left(J_{h}(b)\right)$. Therefore

$$
X_{j} \smile J_{h_{j}}\left(b_{j}\right) \quad \text { for each } j
$$


and hence $Y \smile W$ (the symbol $\smile$ means 'commutes with').

Now the elementary divisors (5) of $W$ are pairwise coprime, since the $b_{j}$ 's are distinct. This implies (see Gantmacher $[1, p .222]$ ) that the first commutant of $W$ coincides with the set of all matrices which are expressible as a polynomial in $W$ over $K$. Therefore $Y$ is expressible as a polynomial in $W$ over $\mathbb{C}$.

If for two matrices $A$ and $B(\neq O)$ in $\mathfrak{M}_{n}(K), B$ is expressible as a polynomial in $A$ over $K$, say $B=p(A)$, then there exists a unique such representing polynomial $p(\lambda)$ of least degree, call it $p_{B, A}(\lambda)$. For by using the Euclidean algorithm in $K[\lambda]$ we can show, first, that for any representing polynomial $p(\lambda)$ of least degree, its degree is less than the degree of the minimal (annihilating) polynomial of $A$; and secondly, that if $p(\lambda)=\alpha_{0} \lambda^{0}+\ldots$ and $q(\lambda)=\beta_{0} \lambda^{\prime}+\ldots$ are two distinct representing polynomials of least degree then, if $\alpha_{0} \neq \beta_{0}$, the polynomial $\left(\beta_{0}-\alpha_{0}\right)^{-1}\left(\beta_{0} p(\lambda)-\alpha_{0} p(\lambda)\right)$ is a representing polynomial of lower degree, which is impossible, while if $\alpha_{0}=\beta_{0}$, then $p(\lambda)-q(\lambda)$ is an annihilating polynomial for $A$, which is also impossible.

Thus in particular there exists a minimal representing polynomial $p_{Y, W}(\lambda)$,

$$
Y=p_{Y, W}(W) .
$$

But then $\left(p_{Y, W}(W)\right)^{m}-f(W)=Y^{m}-f(W)=O$, so $\left(p_{Y, W}(\lambda)\right)^{m}-f(\lambda)$ is an annihilating polynomial for $W$ and hence is a multiple of $w(\lambda)$ : there exists $q(\lambda) \in K[\lambda]$ such that

$$
\left(p_{Y, W}(\lambda)\right)^{m}-f(\lambda)=w(\lambda) q(\lambda) .
$$

This completes the proof of the lemma when $\operatorname{deg}(f) \geqslant 1$. When $f(\lambda)$ is a constant $c$ say, we solve (8) by taking $Y=c^{1 / m} I$ and then argue as before.

Lemma 2. With $K=\mathbf{R}$, let $m, n, w, f$ be as in Lemma 1 and suppose in addition to (i) and (ii) that

(iii) all zeros of $w(\lambda)$ are real, so that $w(\lambda) \in \mathbf{R}[\lambda]$; and

(iv) $f(\lambda) \in R[\lambda]$, and $f(b) \geqslant 0$ at every zero $b$ of $w(\lambda)$.

Then there exist polynomials $p(\lambda)$ and $q(\lambda)$ in $R[\lambda]$ such that (1) holds.

Proof: Under these conditions $W$ is a real matrix and its elementary divisors over $R$ are again (5). The matrices $f\left(J_{k}(b)\right)$ in (7) are real, so $f(W)$ in (6) is real. Let $\rho$ be a ray other than the positive real axis, and let $h$ be the branch function which is real and positive on the positive real axis. Then using (iv) it can be verified that the righthand side of (11) is selfconjugate, so each $X_{j}$ is real and $Y$ in (9) is real. The rest of the argument in the proof of Lemma 1 then applies, with $K=\mathbf{R}$.

The theorem follows immediately from the lemmas. 
Corollary 1. If $w(\lambda)$ has only simple zeros then every [f] in $\mathfrak{U}_{w, \mathrm{C}}$ has $m$ th roots of all orders in that algebra.

COROLLARY 2. The identity coset [1] has $m$ th roots of all orders $m$, in $\mathfrak{U}_{w, C}$ and in $\mathfrak{U}_{w, \mathbf{n}}$, for every choice of polynomial $w(\lambda)$.

We remark that in Lemma 1 the polynomial $p(\lambda)$ satisfies the same conditions as $f(\lambda)$; in Lemma 2, one of $\pm p(\lambda)$ does so.

In each lemma the proof obtains the sought power root $p(\lambda)$ as the representing polynomial of least degree of a particular matrix root of $f(W)$, for a particular matrix $W$ constructed from $w(\lambda)$. The power root is far from being unique; see Examples 3 and 4 below.

The conditions in the theorem are sufficient, not necessary; this is shown in Example 3. But the conditions may not be omitted from the theorem; see Examples 1 and 2.

For any case of $[p]^{m}=[f]$ there are unique polynomials $p_{0}(\lambda)$ and $f_{0}(\lambda)$ in these cosets respectively with degrees less than $n$; necessarily $p_{0}(\lambda)=p_{Y, W}(\lambda)$. Writing $s:=\operatorname{deg}\left(p_{0}\right)$ we have

$$
\frac{n}{m} \leqslant s<n \text { if } q(\lambda) \neq 0, \quad s<\frac{n}{m} \text { if } q(\lambda)=0
$$

In (15) if $f(\lambda)=f_{0}(\lambda)$ we have

$$
\operatorname{deg}(q)=s m-n \text { or } O
$$

EXAMPLES: For low values of $m$ and $n$ and given polynomials $w(\lambda)$ and $f(\lambda)$, one may look for power roots by substituting unknown polynomials $p(\lambda)$ and $q(\lambda)$ in equation (1), assuming minimal degrees, and attempting to solve the resulting nonlinear equations in the coefficients.

1. Take $w(\lambda)=\lambda^{2}(\lambda-1), f(\lambda)=\lambda(\lambda-2), m=2$. Here $f(\lambda)$ has a zero at a multiple zero of $w(\lambda)$. We find that no square root of $[f]$ exists. Thus the condition (ii) in Lemma 1 and the theorem cannot be omitted.

2. Take $w(\lambda)=\lambda(\lambda-2), f(\lambda)=\lambda-1, m=2$. Here all conditions of Lemma 2 are satisfied except that $f(0)<0$ at a zero 0 of $w(\lambda)$. We find that there exists no square root $[p]$ in $\mathfrak{U}_{w, \mathbf{2}}$.

3. Take $w(\lambda)=\lambda(\lambda-1)^{2}, f(\lambda)=(\lambda-1)^{2}, m=2$. Again (ii) fails, but in this case equation (1) has infinitely many solutions with $\operatorname{deg}(p)<n=3$, namely those 
indicated in the table

$$
\begin{array}{cc}
p(\lambda) & q(\lambda) \\
a \lambda^{2}-(a+1) \lambda+1 & a^{2} \lambda-2 a, \\
a \lambda^{2}-(a-1) \lambda-1 & a^{2} \lambda+2 a,
\end{array}
$$

where $a$ is arbitrary in $\mathbb{C}$ (or $\mathbb{R}$ ). The table includes all solutions. Distinct polynomials $p(\lambda)$ determine distinct square roots $[p]$ in $\mathfrak{U}_{w}$, so this $[f]$ has infinitely many square roots in $\mathfrak{U}_{w, \mathbb{C}}$, indeed in $\mathfrak{U}_{w, \mathbb{B}}$. The example also shows the non-necessity of condition (ii).

4. Take $w(\lambda)=(\lambda-a)(\lambda-b)(\lambda-c), f(\lambda)=\lambda, m=2$.

Let $\tau_{1}, \tau_{2}, \tau_{3}$ denote the three elementary symmetric functions on the numbers $a^{1 / 2}, b^{1 / 2}, c^{1 / 2}$, where $a^{1 / 2}$ is chosen to be either one of the two complex square roots of $a$, and likewise for $b^{1 / 2}$ and $c^{1 / 2}$. There are 8 square roots of $f(\lambda) \bmod w(\lambda)$, namely all possible polynomials of the form

with

$$
\begin{gathered}
p(\lambda)=\left(\lambda^{2}+\left(\tau_{2}-\tau_{1}^{2}\right) \lambda-\tau_{1} \tau_{3}\right)\left(\tau_{3}-\tau_{1} \tau_{2}\right)^{-1}, \\
q(\lambda)=\left(\lambda-\tau_{1}^{2}\right)\left(\tau_{3}-\tau_{1} \tau_{2}\right)^{-1},
\end{gathered}
$$

provided $\Delta:=\tau_{3}-\tau_{1} \tau_{2}$ does not vanish. Now $\Delta=0$ if and only if one of $b^{1 / 2}+c^{1 / 2}$, $c^{1 / 2}+a^{1 / 2}, a^{1 / 2}+b^{1 / 2}$ vanishes; hence $\Delta \neq 0$ if $a, b, c$ are distinct.

Suppose $a \neq b=c \neq 0$. We can still ensure that $\Delta \neq 0$ by choosing $b^{1 / 2}=c^{1 / 2}$, and so obtain a square root $[p]$ of $[f]$. However, there are now only 4 distinct square roots.

Suppose $a \neq b=c=0$, so that condition (ii) is violated. In this case $\Delta=0$ and indeed there exists no square root of $[f]$.

\section{REFERENCES}

[1] F.R. Gantmacher, Theory of matrices, Vol 1 (Chelsea, New York, 1960).

[2] E. Hille and R.S. Phillips, Functional analysis and semi-groups (Amer. Math. Soc. Coll. Publ. 31, Providence, 1957). 